\title{
Article
}

\section{Geomagnetic Induced Current Data Generation and Analysis in Power System Installations}

\author{
Chijioke Ejimofor , James Eke, Innocent Eneh. \\ 1 Enugu State University of Science and Technology, Enugu \\ * Correspondence: ejimofor.chijioke@gmail.com; Tel.: +2347045699965
}

\begin{abstract}
Geomagnetic induced current (GIC) is a ground end manifestation associated with the space weather perturbations that should be greatly taken into account by the society. Although the GICs implication to the power system is not regular, it can cause large scale of system failure. In equatorial, the power system is considered safe since the most intense of geomagnetic storm happened in high latitude. However, the internal damage due to GICs which finally led to the South African power system failure has totally changed the normal perception. Therefore, a preliminary investigation on the GICs activity in equatorial region is performed to understand the space weather impact to the power system. Time derivative of the horizontal magnetic field component $(\mathrm{dB} / \mathrm{dt})$ is done to indicate the GICs activity value based on Faraday's law. All the reported power failures are compiled to produce the threshold value of $\mathrm{dB} / \mathrm{dt}$, which possibly cause the harmful effect to the system. Then, $\mathrm{dB} / \mathrm{dt}$ analysis is extended to show the pattern of GICs activity in function of magnetic latitude and local time. The results reveal that power network in equatorial region has possibly suffered by GIC. Plus, high number of intense GIC activity in this region occurred during dayside.
\end{abstract}

Keywords: Geomagnetic induced current (GIC); Magnetic field component (dB/dt) ; Geomagnetic data (GMD) ; Corona mass ejections (CMEs) ;Electric field (E)

\section{Introduction}

The space weather perturbation is believed to affect the ground-based technology system in term of theoretical and experimental approached. The space weather perturbation mentioned above is the geomagnetically induced current (GIC). These GIC flow into the transformer architecture of the bulk power system through the winding where the current in the winding might be increased. More current formed in the winding, more magnetic flux might be generated and the core might be received a partial saturation on the part of the transformer. The partial saturation is believed to damage the working principle of the transformer (Fiori, Boteler, and Gillies 2014). The AC waveform might be misinterpreted, the protective relays might be desperation and drives to trip out the power transmission line. More so, affected by that, the stability problem, the voltage drop, also the power blackout moment is possible to occur. Rapidly mentioned the famous event in 1989 which is the Hydro - Quebec power transmission blackout and the society suffered for nine hours without electricity (Kasran et al. 2019)., Jusoh, M. H., Yoshikawa, A., \& Radzi, Z. M. (2018). The stimulation of the bulk power system enhances the consideration of awareness to identify to what extent the GIC can be impactful to the power transmission system (Ngwira et al. 2013). The rate of the change of the total magnetic field (dB/dt) normally used to identify the GIC level at high latitude region (Pulkkinen et al. 2017). The magnitude of $\mathrm{dB} / \mathrm{dt}(\mathrm{nT} / \mathrm{min}$ ) exceeding $30 \mathrm{nT}$ was indicated the occurrence of the GIC. The high latitude regions are the one that achieves a high possibility that affected by the GIC event due to the auroral electrojet current as a driving mechanism. Previously, the $\mathrm{dB} / \mathrm{dt}$ value during Hydro - Quebec power transmission is approximately $479 \mathrm{nT} / \mathrm{min}$. Some of GIC cases, there was produced not only occurring due to the substorm, but the sudden impulse (SI) and the storm sudden commencement (SSC) also encourage the GIC to occur M. D., Miller, M, \& Wilkinson, P. (2013), Goldthorpe, P, \& Smith, E. A. (2012). The 
authors Kappenman, J. G. (2006) bring some information about the $\mathrm{dB} / \mathrm{dt}$ value are not depending on the substorm as a single caused, but the SI event and the SSC had prepared the evidence that the value of $\mathrm{dB} / \mathrm{dt}$ can still be high enough to penetrate in the power transmission system. This has been discussed by a great number of authors in literature, they proposed a model on how the ionospheric current penetrates in the lithosphere by applying the concept of Lorentz force. It has been reported that substations situated at the ends and at the corners of long transmission lines are prone to maximum GIC magnitudes Magnetic Components R. Ebora and N. Servando, (2009). These are the substations which are only connected to one substation on one end and a transmission line on the other end. However, a number of the study was done in a region close to the auroral zone and may not necessarily agree with regions far from the auroral zone. An investigation was done on the Namibian network and reported that south northerly lines appeared to be more susceptible to GICs The method had been used previously is by determining the time derivative of the horizontal component as conducted by P. J. Cilliers and C. T. Gaunt (2008), , R. Pirjola and A. Viljanen (2007) to determine the GIC phenomena. Meanwhile, other studies are done by calculation approached (Mohd Anuar et al. 2019). In addition, the on-site measurement that needs an equipment that must be conducted under the transmission line that operated with high voltage also had been used by (Piersanti et al. 2019). However, the space weather perturbation that drives GIC during the descending, peak, and ascending phases of the solar cycle is still questionable due to the different mode of solar activity.

Traditionally, transmission lines protection has been done with the aid of relays. From the earliest types which were manually actuated to the present day where highly automated relays are commonly being used in numerous ways. The optimal coordination of primary and backup over-current relays (OCRs) scheme has become one of the main challenges in modern MV-distribution networks (DN) owing to the multi-looped structure and the increased penetration of interment nature-DGs with large-share capacities. The changeable operational topologies of microgrids in modern DN have increased the computational burden to obtain the optimal setting of OCRs. The discrimination time of OCRs scheme may be no longer sufficient or even unmanageable to provide reliable performance in the islanded and grid-connected operational modes. The numerous previously reported optimization techniques employed for OCR coordination may have some limitations/drawbacks regarding OCRs coordination and their framework. Therefore, this study provides a well-defined analytical examination of the limitations in the tripping characteristics of manufactured OCRs. A new constraint taking into consideration maximum plug setting multiplier (PSM) was proposed to improve the optimization techniques. Moreover, enhance the complementary with industrial OCRs tripping characteristics with the optimization techniques in term of coordination time interval (Boteler and Pirjola 2017)

This work focuses on the transmission aspect of the electrical power value chain and considers primarily the protection of the system during faulty conditions of either overcurrent type or ...with the underlying goal of mitigation. When simulating GIC in power systems one needs to approximate field strength of the geoelectric field at injection points, such as grounded transformers. It has been shown (Viljanen et al., 2004) that one can accurately estimate the geoelectric field components from the strength of the geomagnetic field and knowledge of the local ground conductivity. This is of practical interest since the magnetic field fluctuations are easier to measure than the actual geoelectric field strength. Here $x$ and $y$ is in the horizontal plane of the ground and the $x-$ axis is northward pointing and the y-axis is eastward pointing (Zhao and Crossley 2020). Therefore, this study aims to observe and estimate the value of GIC by designing a GMD detection framework. 


\section{Materials and Methods}

Having completed the review of the state of the art in Chapter two, certain unresolved challenges and gaps were identified whose solution have a potential for improving the mitigation of GIC on power system installations. Specifically where GMD have their sources in space weather related incidences which cause GIC that have been shown in section 3.2 to adversely affect the behavior of power systems, sometimes with catastrophic effects. During a GMD event, GIC may cause half-cycle saturation in certain high voltage power transformers, overheating (causes damage especially in older equipment), produce harmonics, increased reactive power requirements, protective power system mis-operation, loss of reactive power sources (static var, capacitors can be tripped) and potential for system collapse- blackouts.

\subsection{Identification of the GIC functional expression from the acquired data}

Fundamentally, the GIC phenomena is an electromagnetic phenomena. Therefore, it is guided by Maxwell's laws thus:

$$
\begin{aligned}
& \nabla \cdot E=\frac{\rho}{\varepsilon_{0}} \\
& \nabla \cdot B=0 \\
& \nabla x E=-\partial B / \partial t \\
& \nabla x B=\mu_{0} j+\mu_{0} \varepsilon_{0} \partial E / \partial t
\end{aligned}
$$

Maxwell's equations are (microscopically) always valid. However, charges and currents are macroscopically usually divided into different types, and additional fields are introduced. The electric $\mathrm{D}$ field is related to $\mathrm{E}$ and the magnetic $\mathrm{H}$ field is related to $\mathrm{B}$ by constitutive equations, which are usually assumed to be linear and simple as follows:

$$
\begin{aligned}
& D=\varepsilon_{0} E \\
& H=B / \mu_{0}
\end{aligned}
$$

where $\mu$ and $\varepsilon$ are the permeability and permittivity of the medium. The usual assumption in connection with geo-electromagnetic studies is that $\mu=\mu 0$. Macroscopically, we can write Maxwell's equations similarly to (2)-(2.5) but $\mu 0$ and $\varepsilon 0$ are replaced by $\mu$ and $\varepsilon$, and the charge density $p=$ pfree and the current density $j=$ jfree should only refer to charges moving freely in the medium. An additional constitutive equation needed in the geophysical part is the Ohm's law that relates jfree and $\mathrm{E}$

$$
j_{\text {free }}=\sigma E
$$

Where $\sigma$ is the conductivity of the medium. What is still required for solving the geophysical part are the continuity conditions that enable moving from one medium to another. Usually we utilize the continuity of the tangential components of the $\mathrm{E}$ and $\mathrm{H}$ fields when moving across a boundary (Pirjola. R, 2012). The GIC is usually computed from the electric and magnetic field data using the formulas:

$$
E_{x}(w)=\frac{Z(w) B_{y}(w)}{\mu_{0}},
$$




$$
\begin{aligned}
& -E_{y}(w)=\frac{Z(w) B_{x}(w)}{\mu_{0}} \\
& \frac{d B}{d t}=\left(\left(\frac{d B x}{d t}\right)^{2}+\left(\frac{d B y}{d t}\right)^{2}\right)^{1 / 2}
\end{aligned}
$$

The resulting GIC has been calculated as a function of the various electric field components by the formula

$$
G I C=a E x(t)+b E y(t)+\varepsilon(t)
$$

where (Ex, Ey) are the horizontal components of the geoelectric field and $(a, b)$ the system parameters. $(a, b)$ that depend on the topology and electrical characteristics of the conductor system under investigation can be derived for individual locations by using information about the full conductor system(Pulkkinen et al. 2013) parameters from GIC and ground magnetic field observations (Pulkkinen et al., 2007). The linear relation in Eq. (2.10) has been shown in numerous studies to hold to a good approximation in many situations of interest (Pulkkinen et al(Pulkkinen et al. 2013), 2006; Ngwira et al., 2008; Pulkkinen et al., 2010). The typical values for $(\mathrm{a}, \mathrm{b})$ are in the range between $0-200 \mathrm{~A} \bullet \mathrm{km} / \mathrm{V}$ (Pulkkinen et al., 2008), and references therein.

\section{Results}

The GIC expression which is used for computation is made possible by utilizing the measured magnetic field data from the center for atmospheric research (CAR) magnetometer station, Abuja, Nigeria. The resulting electric field values were also computed in the axes of the earth's plane (Ex and Ey) for this and stored accordingly in an excel file .Some of the obtained results are presented in this section.

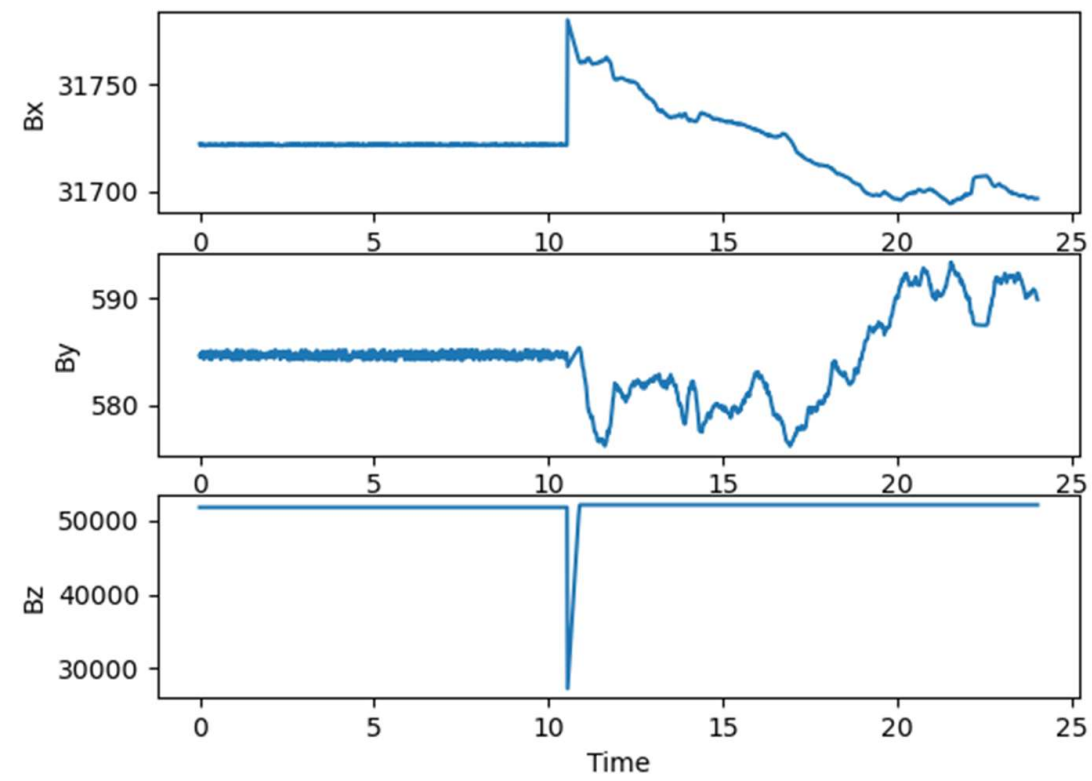

Figure 1. Plot of Bx,By, and Bz with respect to time

The next set of results show the changes in the magnetic field with time or magnetic field derivative 


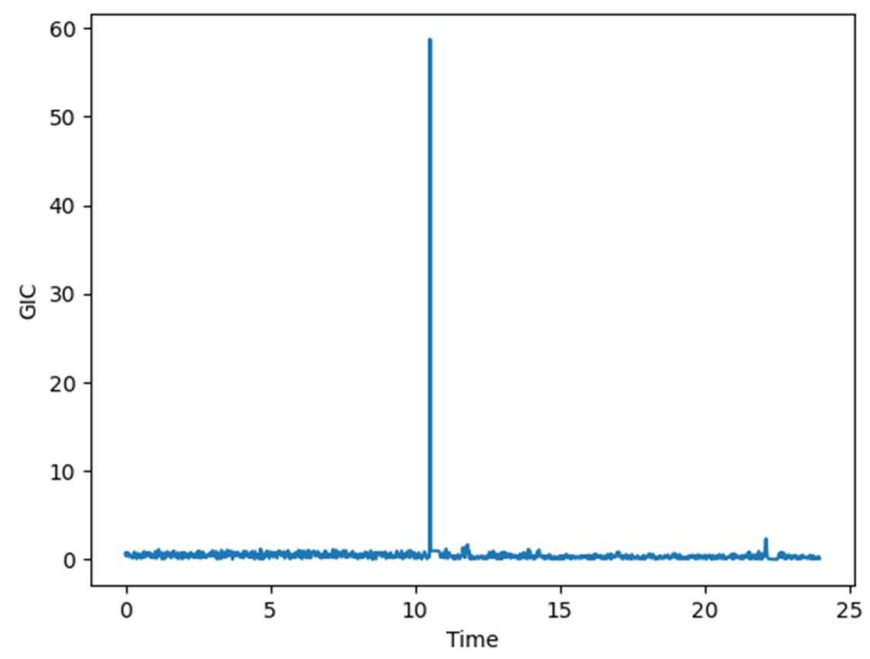

Figure 2. Plot of GIC with respect to time

Finally the first GIC computation showing the induced currents as a function of the changes in magnetic field is given in Figure 2 above. Using the electric field equations for GIC computation, the following plots were obtained with the parameter set given in table 1 below

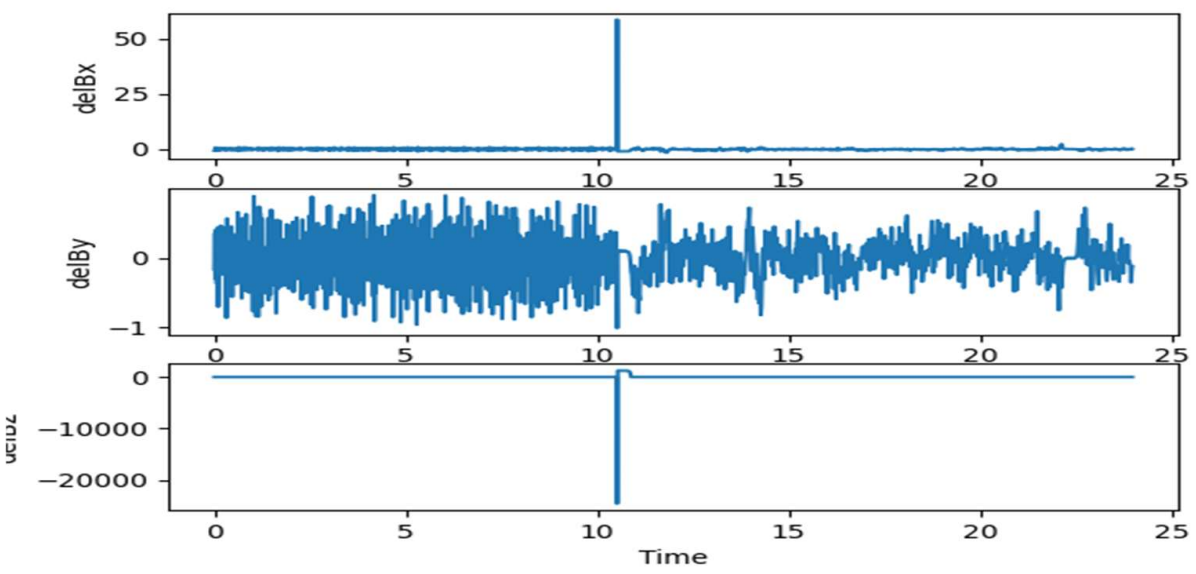

Figure 3. Shows electric field component in the earth's plane as a function of time

Table 1. Plot parameters

\begin{tabular}{lllll}
\hline $\mathrm{s} / \mathrm{n}$ & Z_im & $\mathrm{a}$ & $\mathrm{B}$ & Gaussian noise \\
\hline 1 & 0.01 & 0.5 & 0.717 & 0.00987564 \\
\hline
\end{tabular}

Figure 4 is renders the Electric field component in the earths plane as a function of time. The resulting GIC which was computed from the electric field values and using the parameters in table 1 is shown in figure 5 below. GIC computation result from the electric field obtained from the cost function 


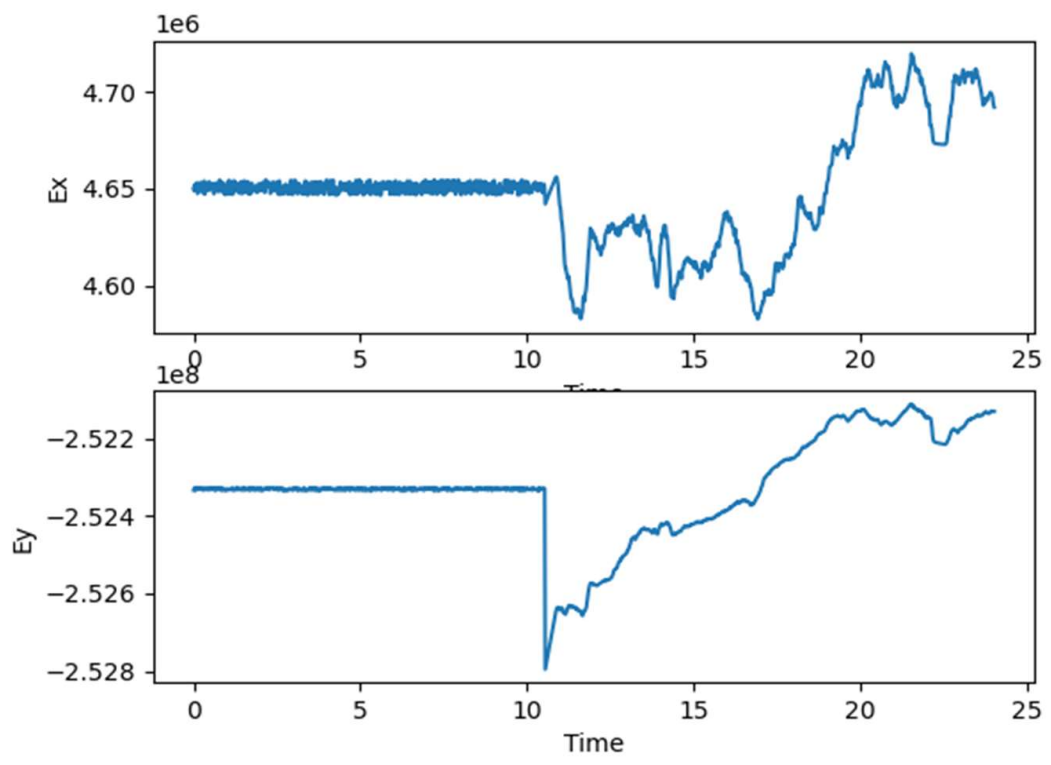

Figure 4. Electric field component in the earth's plane as a function of time

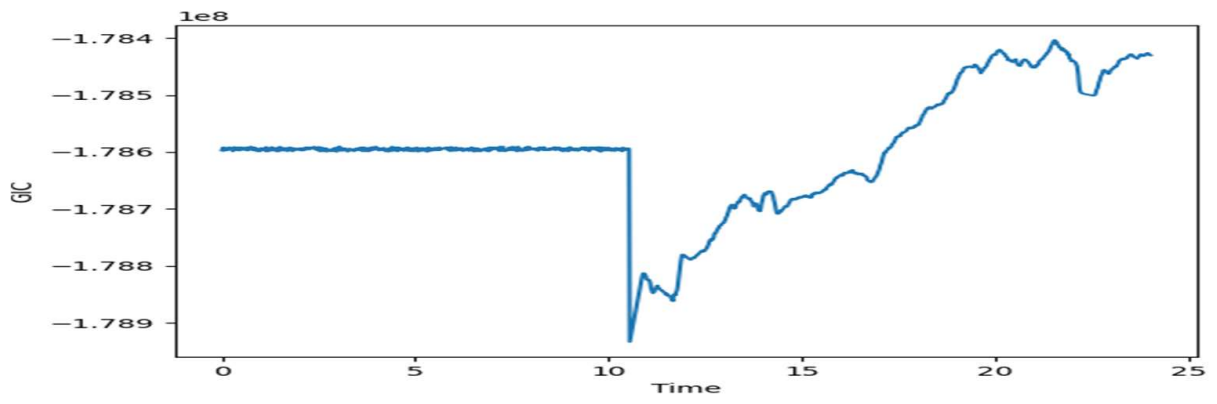

Figure 5. GIC plot from the conditioned electric field components

\section{Discussion}

Geomagnetic data has been acquired from the center for atmospheric sciences (CAR) Abuja. This data was sourced with the aid of a magnetometer instrument which is part of a network of magnetometers managed by CAR and engaged in data collection around the equatorial region. The collected data is saved on a desktop computer or laptop as $\mathrm{Mi}$ crosoft excel spreadsheet files using formats that are amenable to further data manipulation. The data which was used has been saved as an excel comma separated value (csv) file, having appropriate headers and data arranged in row-column format. The duration of data collected and used was for a period of twenty four hours (one day). The sample excel file will be described in terms of its row-columns size as being 1440 rows and 8 columns. The columns are all label in headers which describe the type of data being captured. The primary column names consist of timestamps, number of samples, date of the year, multi-axis GIC data and lastly quality control information. Of interest to this research is the GIC data which has been captured as a triplet of values representing the magnetic field strength in the $x, y$ and $z$ axes. This data is labeled in the header as Bx, By, Bz. Performing some data wrangling on the obtained data, it is necessary to ensure that the data is devoid of any elements that might prevent smooth training and analysis. Missing data samples and not-a-number (nan) entries are also processed to obtain a more representative dataset for modeling the GIC phenomena. This has been achieved with the aid of 
Python programming machine learning toolkit and the code listings can be found in the appendix A of this document. All supporting and derived data files are also available. The GIC is a measure of the level of induced current on the earth's magnetic field during the magnetic storm. It is measured as the quantity of charged energy particle in the magnetosphere. GIC will serve as an output to the system. 1440 data point of measurement for output data, with an hour sampling interval, recorded on a magnetometer was used for the modeling.

\section{Appendix A}

\section{Python code used in the processing of the Raw data}

import os

import numpy as np

import pandas as pd

import matplotlib.pyplot as plt

import openpyxl

print('All modules were accepted')

cwd $=$ os.getcwd ()

print('debugging current working directory: ',cwd)

csv_file='C: $\backslash \backslash$ Users $\backslash \backslash$ Knowdyn $\backslash \backslash$ Desktop $\backslash \backslash$ Copied $\backslash \backslash$ Services folder $\backslash \backslash$ Ejimofor $\backslash \backslash \mathrm{Ph}$ $\mathrm{d} \backslash \backslash$ Data and Scripts $\backslash \backslash \mathrm{CAR} \_$ABUJA_DATA_New.csv'

car_data_rev='C: $\backslash \backslash$ Users $\backslash \backslash$ Knowdyn $\backslash \backslash$ Desktop $\backslash \backslash$ Copied $\backslash \backslash$ Services folder $\backslash \backslash$ Ejimofor $\backslash \backslash$ Phd $\backslash \backslash$ Data and Scripts $\backslash \backslash$ CAR_ABUJA_DATA_REV.csv'

data_file=open(car_data_rev)

csv_data=pd.read_csv(data_file)

print(type(csv_data))

print('Debug....csv_data can be printed but is commented')

print('Extracting single column from csv_data')

$\mathrm{sn}=$ np.linspace $(1.0,8.0$,num=8)

length_sn=len(sn)

print('debug....length serial number', length_sn)

for row in csv_data:

print('\t',row)

print('\n')

print(' Excel files columns are referenced from column zero and upwards')

column_Bx $=$ csv_data.iloc[:,4]

column_By $=$ csv_data.iloc $[:, 6]$

column_Bz $=$ csv_data.iloc[:,8]

len_bxyz=len(column_Bx)

print('Length of the data: ',len_bxyz,'\n')

column_Bxyz=csv_data.iloc[:,4:10]

print(column_Bxyz)

print(' $\backslash n ')$

count_NaN=0

count $=0$

for ii in range(len_bxyz):

if column_Bx[ii]==-9999.0:

count_NaN=count_NaN+1

elif column_Bx[ii]!=-9999.0:

count $=$ count +1

print('debug.....number of NaN or -9999.0 entries: ',count_NaN) 
print('debug.....number of True entries: ',count)

print('print the values that are not null ONLY')

print('\n')

print('Testing the use of random')

import random

\#\# compute the mean of the true values in column Bx

import statistics

import math

import xlsxwriter

\#\#for ii in range(len(column_Bx[632:1439])):

\#\# trueMeanBx=statistics.mean(column_Bx[632:1439])

\#\# \#trueAvgBx=

\#\#print('The mean value of the available values in Bx: ',trueMeanBx)

\#\#print('Populate the NaN values with new guesses baselined with true mean')

\#\#for ii in range(len_bxyz):

\#\# randVal=random.random()

\# if column_Bx[ii]==-9999.0:

\#\# column_Bx[ii]=trueMeanBx+randVal

\#\# elif column_Bx[ii]!=-9999.0:

\#\# column_Bx[ii]=column_Bx[ii]

\#\#

\#\#storedFile='C: $\backslash \backslash$ Users $\backslash \backslash$ Knowdyn $\backslash \backslash$ Desktop $\backslash \backslash$ Copied $\backslash \backslash$ Services folder $\backslash \backslash$ Ejimofor $\backslash$

$\backslash$ Phd $\backslash \backslash$ Data and Scripts $\backslash \backslash$ CAR_ABUJA_DATA_MANIP.xlsx'

\#\#data_file=open(storedFile)

\#\#revDataBx=pd.ExcelWriter(storedFile,engine='xlsxwriter')

\#\#column_Bx.to_excel(revDataBx,'Sheet1')

\#\#revDataBx.save()

\#\#\#\#\#\#\#\#\#\#\#\#\#\#\#\#\#\#\#\#\#\#\#

\#\#for ii in range(len(column_By[632:1439])):

\#\# trueMeanBy=statistics.mean(column_By[632:1439])

\#\# \#trueAvgBy=

\#\#print('The mean value of the available values in By: ',trueMeanBy)

\#\#print('Populate the NaN values with new guesses baselined with true mean')

\#\#for ii in range(len_bxyz):

\#\# randVal=random.random()

\#\# if column_By[ii]==-9999.0:

\#\# column_By[ii]=trueMeanBy+randVal

\#\# elif column_By[ii]!=-9999.0:

\#\# column_By[ii]=column_By[ii]

\#\#

\#\#storedFile='C: $\backslash \backslash$ Users $\backslash \backslash$ Knowdyn $\backslash \backslash$ Desktop $\backslash \backslash$ Copied $\backslash \backslash$ Services folder $\backslash \backslash$ Ejimofor $\backslash$ $\backslash$ Phd $\backslash \backslash$ Data and Scripts $\backslash \backslash$ CAR_ABUJA_DATA_MANIP.xlsx'

\#\#data_file=open(storedFile)

\#\#revDataBy=pd.ExcelWriter(storedFile,engine='xlsxwriter')

\#\#column_By.to_excel(revDataBy,'Sheet1')

\#\#revDataBy.save()

\#\#\#\#\#\#\#\#\#\#\#\#\#\#\#\#\#\#\#\#\#\#\#\#\#\# 
\#\#for ii in range(len(column_Bz[632:1439])):

\#\# trueMeanBz=statistics.mean(column_Bz[632:1439])

\#\# \#trueAvgBz=

\#\#print('The mean value of the available values in By: ',trueMeanBz)

\#\#print('Populate the NaN values with new guesses baselined with true mean')

\#\#for ii in range(len_bxyz):

\#\# randVal=random.random()

\# if column_Bz[ii]==-9999.0:

\#\# column_Bz[ii]=trueMeanBz+randVal

\#\# elif column_Bz[ii]!=-9999.0:

\#\# column_Bz[ii]=column_Bz[ii]

\#\#

\#\#storedFile='C: $\backslash \backslash$ Users $\backslash \backslash$ Knowdyn $\backslash \backslash$ Desktop $\backslash \backslash$ Copied $\backslash \backslash$ Services folder $\backslash \backslash$ Ejimofor $\backslash$ $\backslash$ Phd $\backslash \backslash$ Data and Scripts $\backslash \backslash$ CAR_ABUJA_DATA_MANIP.xlsx'

\#\#data_file $=$ open(storedFile)

\#\#revDataBz=pd.ExcelWriter(storedFile,engine='xlsxwriter')

\#\#column_Bz.to_excel(revDataBz,'Sheet1')

\#\#revDataBz.save()

\#\#\#\#\#\#\#\#\#\#\#\#\#\#\#\#\#\#\#\#\#\#\#\#\#\#\#

\#\# Now I want to plot the various Magnetic field values for Bx, By, Bz

\# note that the dataframe being referenced by csv_data was read previously

column_Bx_ed $=$ csv_data.iloc[:,5]

column_By_ed $=$ csv_data.iloc[:,7]

column_Bz_ed $=$ csv_data.iloc[:,9]

"

Do some extra analysis with the derived data

1. I will be computing the first set of GIC data from Bx and By

"'"'!

len_data_ed=len(column_Bx_ed)

print('length of data to work with: ',len_data_ed)

count $=0$

column_DelBx=csv_data.iloc[:,12]

column_DelBy=csv_data.iloc[:,13]

column_DelBz=csv_data.iloc[:,14]

column_IC=csv_data.iloc[:,15]

\#\#for ii in range(len_data_ed-1):

\#\# column_DelBx[ii]=column_Bx_ed[ii+1]-column_Bx_ed[ii]

\#\# column_DelBy[ii]=column_By_ed[ii+1]-column_By_ed[ii]

\#\# column_DelBz[ii]=column_Bz_ed[ii+1]-column_Bz_ed[ii]

\#\# column_IC[ii]=math.sqrt((column_DelBx[ii] ${ }^{*}$ column_DelBx[ii])+(column_DelBy[ii] ${ }^{*} \mathrm{C}$

olumn_DelBy[ii]))

\# count=count +1

\#\#storedFile='C: $\backslash \backslash$ Users $\backslash \backslash$ Knowdyn $\backslash \backslash$ Desktop $\backslash \backslash$ Copied $\backslash \backslash$ Services folder $\backslash \backslash$ Ejimofor $\backslash$

$\backslash$ Phd $\backslash \backslash$ Data and Scripts $\backslash \backslash$ CAR_ABUJA_DATA_MANIP.xlsx'

\#\#dataIC=pd.ExcelWriter(storedFile,engine='xlsxwriter')

\#\#column_IC.to_excel(dataIC,'Sheet1')

\#\#dataIC.save() 


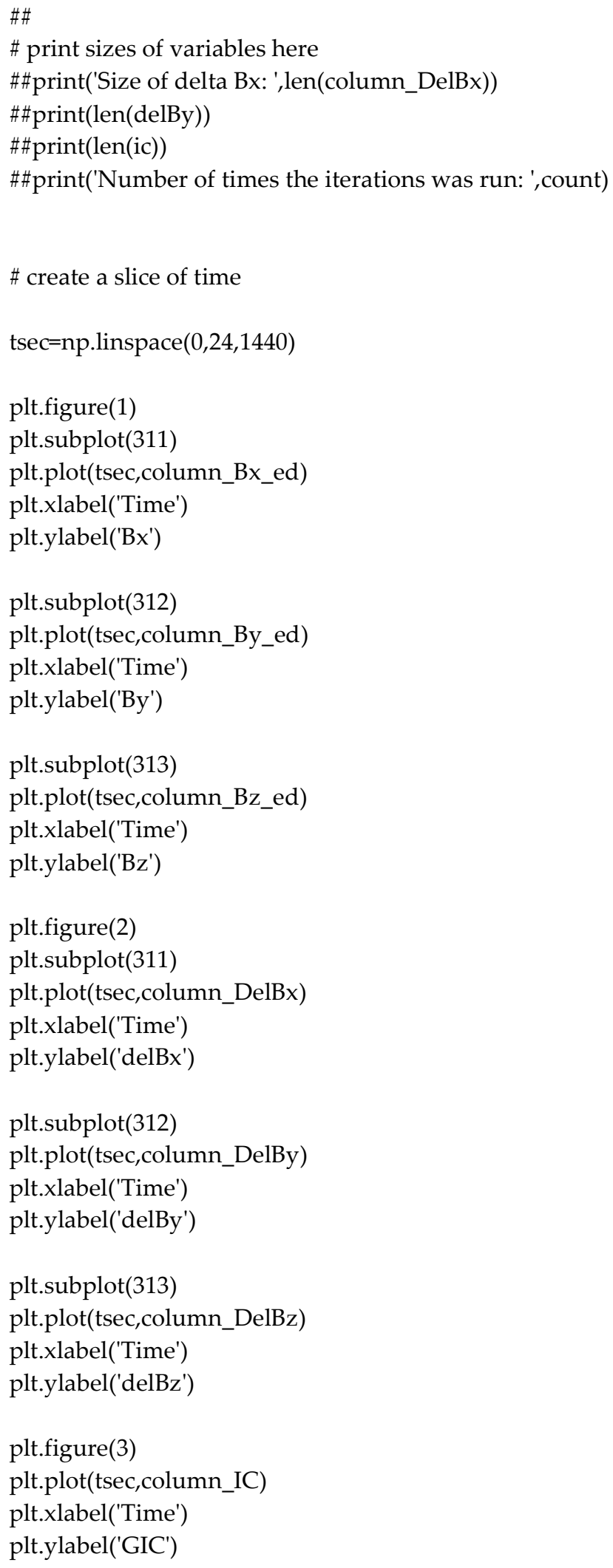


plt.show()

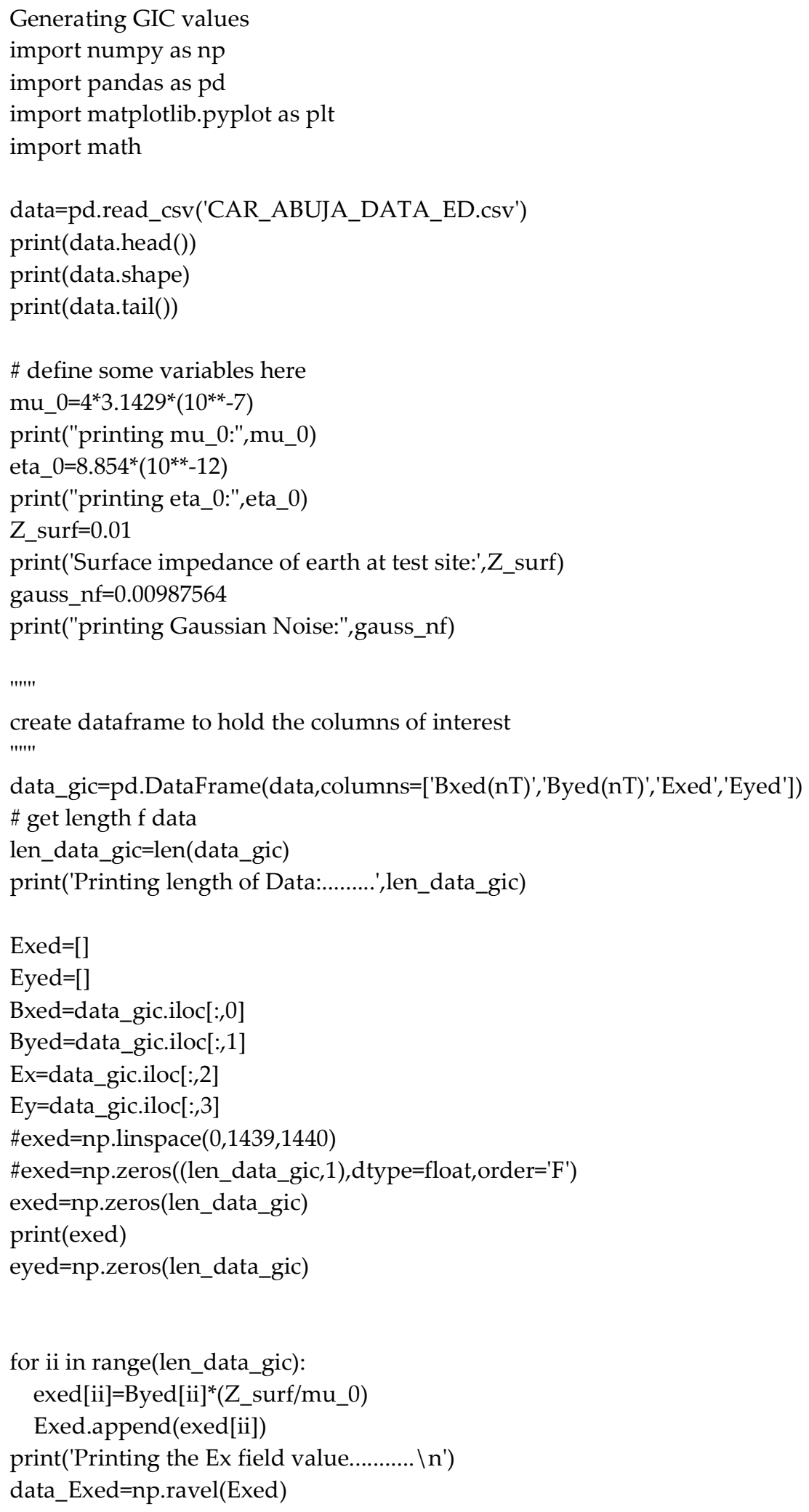




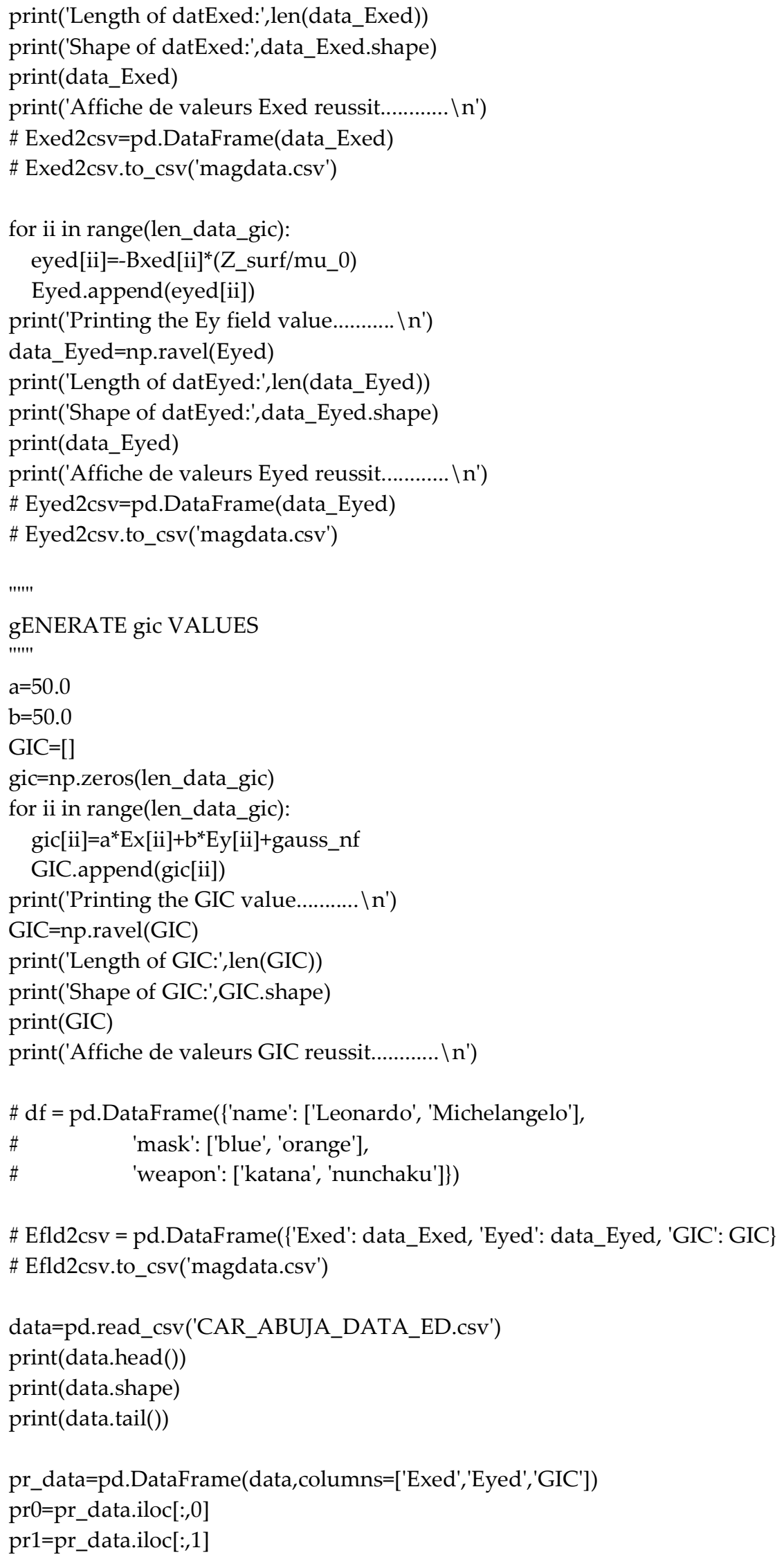




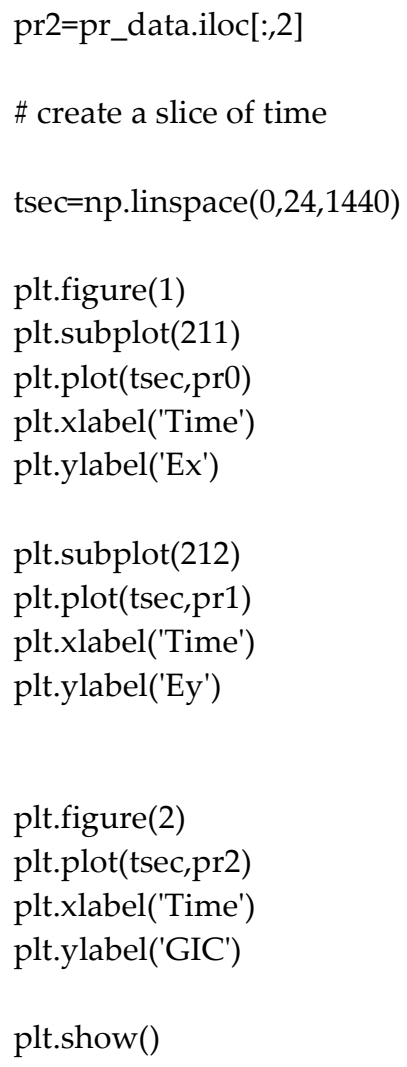

Determining the constants of the model identification

"'"'"

Without step test identification

"'"'"

from gekko import GEKKO

import pandas as $\mathrm{pd}$

import matplotlib.pyplot as plt

\# load data and parse into columns

\#dataPtr='MOdelData.txt'

dataPtr='ModelData.csv'

data $=$ pd.read_csv(dataPtr)

$\mathrm{t}=$ data['Sample']

$\mathrm{u}=$ data[['Exed','Eyed']]

$\# \mathrm{y}=$ data[['T1','T2']]

$\left.\mathrm{y}=\operatorname{data}\left[{ }^{\prime} \mathrm{GIC}\right]\right]$

\# generate time-series model

$\mathrm{m}=\mathrm{GEKKO}$ (remote=False) \# remote=True for MacOS

print('Dislay Time series model .$\backslash \mathrm{n}$ ')

$\operatorname{print}\left({ }^{\prime * * * * * 1}, \mathrm{~m}\right)$

print('COnstants: ',m._constants) 
$\operatorname{print}\left(' \backslash n^{\prime}\right)$

\# system identification

na $=4$ \# output coefficients ....originally had 2 as value

$\mathrm{nb}=4$ \# input coefficients ....originally had 2 as value

$\mathrm{yp}, \mathrm{p}, \mathrm{K}=\mathrm{m} . \operatorname{sysid}(\mathrm{t}, \mathrm{u}, \mathrm{y}, \mathrm{na}, \mathrm{nb}$, diaglevel=1)

APMonitor, Version 0.9.2

APMonitor Optimization Suite

APM Model Size

Each time step contains

Objects : 3

Constants : 6

Variables : 5780

Intermediates: 0

Connections : 15

Equations : 2887

Residuals : 2887

Number of state variables: 1454

Number of total equations: - 1441

Number of slack variables: - 0

Degrees of freedom $\quad: \quad 13$

$* * * * * * * * * * * * * * * * * * * * * * * * * * * * * * * * * * * * * * * * * * * * * *$

Model Parameter Estimation with Interior Point Solver

$* * * * * * * * * * * * * * * * * * * * * * * * * * * * * * * * * * * * * * * * * * * * * *$

Info: Exact Hessian

$* * * * * * * * * * * * * * * * * * * * * * * * * * * * * * * * * * * * * * * * * * * * * * * * * * * * * * * * * * * * * * * * * * * * * * * * * * * * * *$

This program contains Ipopt, a library for large-scale nonlinear optimization.

Ipopt is released as open source code under the Eclipse Public License (EPL).

For more information visit http://projects.coin-or.org/Ipopt

$* * * * * * * * * * * * * * * * * * * * * * * * * * * * * * * * * * * * * * * * * * * * * * * * * * * * * * * * * * * * * * * * * * * * * * * * * * * * *$

This is Ipopt version 3.10.2, running with linear solver mumps.

Number of nonzeros in equality constraint Jacobian...: 25859

Number of nonzeros in inequality constraint Jacobian.: $\quad 0$

Number of nonzeros in Lagrangian Hessian.............. $\quad 7185$

Total number of variables.............................. $\quad 1454$

variables with only lower bounds:

variables with lower and upper bounds: 
variables with only upper bounds:

Total number of equality constraints.

Total number of inequality constraints. inequality constraints with only lower bounds: inequality constraints with lower and upper bounds: inequality constraints with only upper bounds:
0

0

iter objective inf_pr inf_du $\lg (\mathrm{mu}) \quad|| \mathrm{d}|| \quad \lg (\mathrm{rg})$ alpha_du alpha_pr ls $05.4295945 \mathrm{e}-0024.36 \mathrm{e}+0008.25 \mathrm{e}-003 \quad 0.00 .00 \mathrm{e}+000 \quad-0.00 \mathrm{e}+0000.00 \mathrm{e}+000$ $11.9189719 \mathrm{e}+0006.36 \mathrm{e}-0018.65 \mathrm{e}+001 \quad 2.04 .82 \mathrm{e}+000$ $22.0065154 \mathrm{e}+0006.15 \mathrm{e}-0016.45 \mathrm{e}+001 \quad 3.95 .56 \mathrm{e}+000$ $31.7250885 \mathrm{e}+0002.67 \mathrm{e}+0002.22 \mathrm{e}+002 \quad-2.03 .52 \mathrm{e}+000$ $41.6840343 \mathrm{e}+0006.51 \mathrm{e}-0031.23 \mathrm{e}+001 \quad-0.15 .25 \mathrm{e}-001$ $51.3983008 \mathrm{e}+0005.68 \mathrm{e}-0032.63 \mathrm{e}+000 \quad-2.14 .24 \mathrm{e}-001$ $61.0842605 \mathrm{e}+0001.30 \mathrm{e}-0023.01 \mathrm{e}+000 \quad-3.97 .21 \mathrm{e}-001$ $78.2961592 \mathrm{e}-0011.92 \mathrm{e}-0022.34 \mathrm{e}+000 \quad-5.81 .04 \mathrm{e}+000$ $86.7951099 \mathrm{e}-001$ 1.98e-002 1.26e+000 $-7.71 .31 \mathrm{e}+000$ $96.2730538 \mathrm{e}-001$ 1.13e-002 9.10e-001 $-9.51 .31 \mathrm{e}+000$ - 9.90e-001 1.00e+000h 1 - 9.98e-001 2.50e-001h 3 - 9.90e-001 1.00e+000h 1 $0.09 .98 \mathrm{e}-0011.00 \mathrm{e}+000 \mathrm{~h} \quad 1$ $-0.59 .98 \mathrm{e}-0011.00 \mathrm{e}+000 \mathrm{~h} \quad 1$ $-1.01 .00 \mathrm{e}+0001.00 \mathrm{e}+000 \mathrm{f} 1$ $-1.41 .00 \mathrm{e}+0001.00 \mathrm{e}+000 \mathrm{~h} \quad 1$ $-1.91 .00 \mathrm{e}+0001.00 \mathrm{e}+000 \mathrm{~h} \quad 1$ iter objective inf_pr inf_du $\lg (\mathrm{mu}) \quad|| \mathrm{d}|| \quad \lg (\mathrm{rg})$ alpha_du alpha_pr ls $106.3204887 \mathrm{e}-0014.94 \mathrm{e}-003$ 3.63e-001 -11.0 4.35e+000 - $1.00 \mathrm{e}+000$ 2.50e-001h 3 $116.2283585 \mathrm{e}-0011.39 \mathrm{e}-0021.79 \mathrm{e}+000-11.03 .28 \mathrm{e}+000 \quad-1.00 \mathrm{e}+0001.00 \mathrm{e}+000 \mathrm{~h} 1$ 12 6.1319952e-001 1.85e-003 2.17e-001 -11.0 1.26e+000 - $1.00 \mathrm{e}+0001.00 \mathrm{e}+000 \mathrm{~h} 1$ 13 6.1196840e-001 4.24e-005 9.73e-003 -11.0 7.16e-002 $146.1192738 \mathrm{e}-0012.66 \mathrm{e}-008$ 2.26e-006 -11.0 1.94e-003 - $1.00 \mathrm{e}+0001.00 \mathrm{e}+000 \mathrm{~h} 1$ 15 6.1192735e-001 6.44e-015 2.09e-013 -11.0 2.76e-007 - $1.00 \mathrm{e}+0001.00 \mathrm{e}+000 \mathrm{~h} 1$ - $1.00 \mathrm{e}+0001.00 \mathrm{e}+000 \mathrm{~h} 1$

Number of Iterations....: 15

Objective. (scaled) (unscaled) Dual infeasibility......: $\quad 2.0892315655274274 \mathrm{e}-013 \quad 2.0892315655274274 \mathrm{e}-013$ Constraint violation....: $\quad 6.4392935428259079 \mathrm{e}-015 \quad 6.4392935428259079 \mathrm{e}-015$ Complementarity.........: $\quad 9.9999999999999994 \mathrm{e}-012 \quad 9.9999999999999994 \mathrm{e}-012$ Overall NLP error....... $\quad 9.9999999999999994 \mathrm{e}-012 \quad 9.9999999999999994 \mathrm{e}-012$

Number of objective function evaluations $\quad=25$

Number of objective gradient evaluations $\quad=16$

Number of equality constraint evaluations $\quad=25$

Number of inequality constraint evaluations $\quad=0$

Number of equality constraint Jacobian evaluations $=16$

Number of inequality constraint Jacobian evaluations $=0$

Number of Lagrangian Hessian evaluations $\quad=15$

Total CPU secs in IPOPT (w/o function evaluations) = $\quad 5.865$

Total CPU secs in NLP function evaluations $=4.740$

EXIT: Optimal Solution Found.

The solution was found.

The final value of the objective function is $\quad 0.6119273463775022$ 


\begin{tabular}{|c|c|}
\hline Solver & : IPOPT (v3.12) \\
\hline Solution time & : $15.824000000000012 \mathrm{sec}$ \\
\hline Objective & $: \quad 0.6119273463775022$ \\
\hline
\end{tabular}

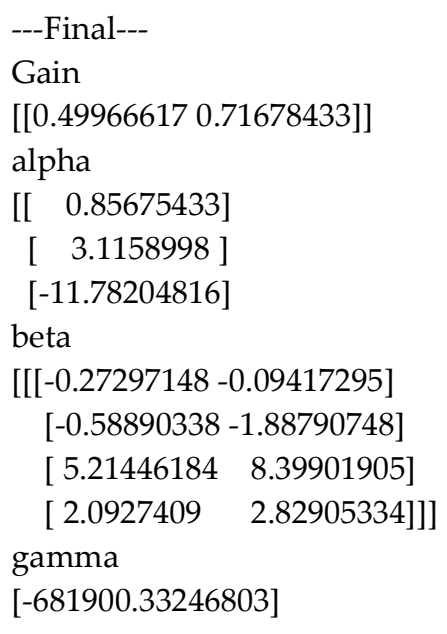

\section{References}

Boteler, D. H., and R. J. Pirjola. 2017. "Modeling geomagnetically induced currents." Space Weather no. 15 (1):258-276. doi: 10.1002/2016SW001499.

Fiori, R. A. D., D. H. Boteler, and D. M. Gillies. 2014. "Assessment of GIC risk due to geomagnetic sudden commencements and identification of the current systems responsible." Space Weather no. 12 (1):76-91. doi: 10.1002/2013SW000967

Kasran, F. A. M., M. H. Jusoh, S. A. E. A. Rahim, and N. Abdullah. 2019. Geomagnetically Induced Currents (GICs) in equatorial region. Paper read at 8th International Conference on System Engineering and Technology, ICSET 2018.

Mohd Anuar, N., F. A. Mohd Kasran, M. Abbas, M. H. Jusoh, S. A. Enche Ab Rahim, N. Abdul Hadi, A. Yoshikawa, and Z. Mohd Radzi. 2019. Assessment of the Geomagnetically Induced Current (GIC) at Low Latitude Region based on MAGDAS Data. Paper read at 1st International Conference on Space Weather and Satellite Application 2018, ICeSSAT 2018.

Ngwira, C. M., A. Pulkkinen, F. D. Wilder, and G. Crowley. 2013. "Extended study of extreme geoelectric field event scenarios for geomagnetically induced current applications." Space Weather no. 11 (3):121-131.

Piersanti, M., S. Di Matteo, B. A. Carter, J. Currie, and G. D'Angelo. 2019. "Geoelectric Field Evaluation During the September 2017 Geomagnetic Storm: MA.I.GIC. Model." Space Weather. doi: 10.1029/2019SW002202.

Pulkkinen, A., E. Bernabeu, A. Thomson, A. Viljanen, R. Pirjola, D. Boteler, J. Eichner, P. J. Cilliers, D. Welling, N. P. Savani, R. S. Weigel, J. J. Love, C. Balch, C. M. Ngwira, G. Crowley, A. Schultz, R. Kataoka, B. Anderson, D. Fugate, J. J. Simpson, and M. MacAlester. 2017. "Geomagnetically induced currents: Science, engineering, and applications readiness." Space Weather no. 15 (7):828-856. doi: 10.1002/2016SW001501.

Pulkkinen, A., L. Rastätter, M. Kuznetsova, H. Singer, C. Balch, D. Weimer, G. Toth, A. Ridley, T. Gombosi, M. Wiltberger, J. Raeder, and R. Weigel. 2013. "Community-wide validation of geospace model ground magnetic field perturbation predictions to support model transition to operations." Space Weather no. 11 (6):369-385. doi: 10.1002/swe.20056

Zhao, Y., and P. Crossley. 2020. "Impact of DC bias on differential protection of converter transformers." International Journal of Electrical Power and Energy Systems no. 115. doi: 10.1016/j.ijepes.2019.105426. 